\title{
Avaliação da flexibilidade de crianças com paralisia cerebral espástica após intervenção fisioterapêutica
}

\section{Assessment of the flexibility of children with spastic cerebral palsy after physiotherapeutic intervention}

\author{
Ana Paula Espindulal; Domingos Emanuel Bevilacqua Júnior?; Mariane Fernandes Ribeiro2; \\ Janaine Brandão Lagel; Ednéia Corrêa de Mello; ; Juliana Beatriz Espindula Raizel³; Vicente de \\ Paula Antunes Teixeira ${ }^{1}$ \\ 1 Programa de Pós-Graduação em Ciências da Saúde - Universidade Federal do Triângulo Mineiro - UFTM. Uberaba, MG - Brasil. \\ 2 Departamento de Fisioterapia - Faculdade Patos de Minas - FPM. Patos de Minas, MG - Brasil. \\ 3 Ensino Médio completo - Escola Estadual Professora Corina de Oliveira. Uberaba, MG - Brasil. \\ Endereço para Correspondência \\ Ana Paula Espindula \\ Disciplina de Patologia Geral, Universidade Federal \\ do Triângulo Mineiro. \\ Rua Frei Paulino, 30 \\ 38025-180 - Uberaba - MG [Brasil] \\ anapaulaespindula@yahoo.com.br
}

\begin{abstract}
Resumo
Introdução: A fisioterapia favorece a flexibilidade e diminue a espasticidade de crianças com paralisia cerebral (PC). Objetivos: avaliar os benefícios da fisioterapia convencional para a flexibilidade a curto e longo prazo de crianças com PC espástica. Métodos: participaram seis pacientes com PC espástica, idade de seis a 14 anos, ambos os gêneros. Foram realizadas 16 sessões de fisioterapia convencional com alongamentos passivos. Para avaliação da flexibilidade, aplicou-se o Teste de Sentar e Alcançar utilizando o Banco de Wells antes e após cada sessão. Para a análise estatística foi utilizado o Software Sigma-Stat ${ }^{\circledR}$ versão 3.5 com significância estatística $(\mathrm{p}<0,05)$. Resultados: houve aumento significativo da flexibilidade da cadeia muscular posterior comparando pré e pós individualmente $(p<0,05)$ e da média pré e pós sessões $(p<0,0001)$. Conclusão: fisioterapia convencial por meio de exercícios de alongamentos passivos melhora a flexibilidade da cadeia muscular posterior de crianças com PC espástica a curto ou a longo prazo.
\end{abstract}

Descritores: Fisioterapia; Paralisia Cerebral; Exercícios de alongamento muscular.

\begin{abstract}
Introduction: Physiotherapy favors flexibility and reduce the spasticity of children with spastic cerebral palsy. Objectives: To evaluate the benefits of conventional physiotherapy to improve the flexibility of the posterior muscle chain in the short and long term of children with spastic cerebral palsy. Methods: participated six patients with a diagnosis of Cerebral Palsy, age of six to 14 years, both genders. There were 16 sessions of conventional physiotherapy with passive stretching. For the assessment of flexibility, the sit and reach test was applied using the Wells's box before and after each session. For statistical analysis, Sigma-Stat ${ }^{\circledR}$ Software version 3.5 was used with statistical significance $(p<0.05)$. Results: There was a significant increase in the flexibility of the posterior muscular chain of the children when comparing pre and post individually $(\mathrm{p}<0.05)$ and the mean before and after the sessions ( $\mathrm{P}<0.0001)$. Conclusion: Conventional physiotherapy through passive stretching exercises improves the flexibility of the posterior muscular chain of children with spastic Cerebral Palsy in the short or long term.
\end{abstract}

Keywords: Physical Therapy Specialty; Cerebral Palsy; Muscle Stretching Exercises. 


\section{Introdução}

O termo Paralisa Cerebral (PC) é reconhecido como uma condição do desenvolvimento neurológico que se caracteriza por alterações ocorridas na primeira infância, as quais persistem por toda a vida, porém de maneira não progressiva $^{1}$. Tais alterações ocasionam desordens permanentes no desenvolvimento quanto ao movimento e à postura, causando incapacidades $^{2}$. Frequentemente as alterações motoras são acompanhadas por distúrbios sensitivos, perceptivos, cognitivos, comunicativos e comportamentais, decorrentes de crises epilépticas ou alterações musculoesqueléticas secundárias ${ }^{1,3}$.

Desta forma, a PC pode ser considerada uma disfunção predominantemente sensoriomotora com alterações no tônus, decorrente de lesões não evolutivas do sistema nervoso central durante a fase de maturação estrutural e funcional envolvendo os períodos pré, peri e pós-natal ${ }^{4}$.

De acordo com variedade das apresentações clínicas, a PC pode ser classificada segundo o foco da lesão encefálica e as manifestações topográficas corporais ${ }^{5}$. As alterações do tônus muscular também são fatores que classificam a PC quanto às desordens do movimento em espástica, discinética e atáxica ${ }^{6}$. As características de locomoção e funcionalidade do indivíduo decorrentes da gravidade do comprometimento neuromotor podem classificar a PC em níveis de I a $\mathrm{V}$, sendo $\mathrm{V}$ o nível com maior comprometimento ${ }^{7}$. A forma mais comum da PC é a forma espástica, também chamada piramidal, e pode ser observada em $88 \%$ dos casos $^{8}$.

Dados epidemiológicos da população brasileira estimam uma incidência de sete para 1000 nascidos vivos ${ }^{9}$, no entanto atualmente não existem dados conclusivos acerca do número de casos dessa população ${ }^{10}$. No Brasil, evidências apontam que a PC é resultado da associação da predisposição genética com os fatores ambientais intra e extrauterinos desencadeantes. As causas que se apresentam com maior prevalência no período intrauterino (pré-natal) são retardo no crescimento com percentil menor que três, doença tireoidiana materna e pré-eclâmpsia grave, enquanto que nos aspectos extrauterinos (perinatais) os eventos intraparto agudo (anóxia cerebral), parto instrumentado e cesariana de emergência (prematuridade) são os fatores mais relevantes ${ }^{11}$. Ações de saúde e educação voltadas a essa população, revelam que as condições socioeconômicas, podem influenciar o desenvolvimento da criança com PC, sendo consideradas preditivos de risco ${ }^{12,13}$.

Como consequência das alterações neuromotoras, a PC pode resultar em incapacidades, ou seja, limitações no desempenho de atividades e tarefas do cotidiano da criança e de sua família que comprometem o desenvolvimento neurosensoriomotor desses indivíduos de acordo com os padrões de normalidade $\mathrm{e}^{14,15}$.

Assim, intervenções que envolvam alongamento têm sido recomendadas como tratamento para a melhora da flexibilidade e da amplitude de movimento articular, tornandose ferramentas importantes em áreas distintas relacionadas à função motora ${ }^{16,17}$. Uma única sessão de alongamento é capaz de proporcionar um aumento da flexibilidade muscular e a amplitude do movimento, de modo imediato, contribuindo, dessa maneira, para melhorar o bem-estar e a qualidade de vida ${ }^{18}$. Neste sentido, a fisioterapia, por meio da cinesioterapia, empregando exercícios de alongamento, pode oferecer ao paciente com PC melhora na flexibilidade e espasticidade ${ }^{19}$.

Portanto, o objetivo geral deste estudo é avaliar os benéficos da fisioterapia convencional para a melhora da flexibilidade da cadeia muscular posterior em crianças com PC espástica.

\section{Materiais e métodos}

O presente estudo é descritivo, analítico e longitudinal. De acordo com os preceitos adotados em pesquisas com seres humanos, a pesquisa foi aprovada pelo Comitê de Ética em Pesquisa conforme o protocolo 2686. 


\section{Participantes}

Foram analisados seis pacientes com diagnóstico de PC devidamente matriculados em uma instituição especializada no atendimento de crianças com deficiências, com idade média $9,83( \pm 3,25)$ anos, sendo três do gênero feminino e três do masculino. Quanto à classificação topográfica da PC, quatro crianças apresentavam quadriparesia espástica e duas diparesia espástica (Tabela 1). Os critérios para seleção dos participantes foram baseados na análise prévia dos prontuários da instituição. Desta forma, foram considerados como critérios de inclusão a necessidade da assiduidade na instituição, apresentarem diagnóstico clínico de PC do tipo espástica, faixa etária entre seis e 14 anos, ambos os gêneros e encaminhamento para fisioterapia. No entanto, não foram incluídas crianças que apresentassem síndromes associadas e que estivessem realizando equoterapia ou outra terapia física. Foram excluídos do estudo, os indivíduos com alterações comportamentais e/ou intelectuais que comprometessem o desempenho das atividades estabelecidas no protocolo de tratamento, assim como duas faltas consecutivas.

Tabela 1: Caracterização dos participantes

\begin{tabular}{c|c|c|c|c}
\hline Paciente & Idade & Gênero & $\begin{array}{c}\text { Classificação } \\
\text { da PC } \\
\text { (Tônus } \\
\text { muscular) }\end{array}$ & $\begin{array}{c}\text { Classificação } \\
\text { da PC } \\
\text { (Topográfica) }\end{array}$ \\
\hline 1 & 14 anos & Masculino & Espástica & Diparética \\
\hline 2 & 11 anos & Feminino & Espástica & Quadriparética \\
\hline 3 & 6 anos & Masculino & Espástica & Diparética \\
\hline 4 & 6 anos & Feminino & Espástica & Quadriparética \\
\hline 5 & 10 anos & Masculino & Espástica & Quadriparética \\
\hline 6 & 12 anos & Feminino & Espástica & Quadriparética \\
\hline
\end{tabular}

Fonte: Do autor, 2018.

\section{Metodologia}

A avaliação da flexibilidade e as sessões de fisioterapia foram realizadas no setor de fisioterapia da instituição, em um local apropriado para o desenvolvimento da pesquisa, contendo instrumentos necessários (tablado e banco de Wells) para aplicação dos exercícios propostos durante o atendimento fisioterapêutico. A rotina de atendimento dos pacientes contou com a colaboração da equipe de profissionais da instituição e proponentes da pesquisa.

Para a avaliação da flexibilidade foi empregado o teste de sentar e alcançar utilizando o banco de Wells, realizada de maneira passiva. Sendo assim, o avaliador conduziu o movimento até a percepção da resistência muscular imposta pela limitação do alongamento. O teste foi aplicado anteriormente à primeira sessão e ao final da $16^{\mathrm{a}}$ sessão.

O teste consiste na avaliação da cadeia muscular posterior de tronco e membros inferiores. Inicialmente, o indivíduo foi posicionado sentado em frente ao banco com os joelhos estendidos, apoiando totalmente a planta dos pés na face anterior do banco. Posteriormente, foi solicitado que estendesse os braços e cotovelos, sobrepondo uma mão a outra e flexionando o tronco para frente até o seu limite, sem que ocorresse alteração quanto ao posicionamento inicial. É aconselhável realizar três medidas do teste em cada avaliação para que posteriormente, sejam obtidos valores mais fidedignos por meio do cálculo das suas médias ${ }^{20,21}$. Desta forma, no estudo em questão foram coletadas as três medidas para cada avaliação. De acordo com a média dos resultados, a flexibilidade foi classificada segundo a distância alcançada em centímetros $(\mathrm{cm})$, como fraca quando o participante alcançasse até $11 \mathrm{~cm}$, regular para o alcance de 12 e $13 \mathrm{~cm}$, média entre 14 e $18 \mathrm{~cm}$, boa para valores de 19 a $21 \mathrm{~cm}$ e excelente quando atingisse acima de $22 \mathrm{~cm}$.

Para as sessões de fisioterapia foi estabelecido um protocolo composto por seis exercícios que consistiam no alongamento da cadeia muscular de membros inferiores, conforme descritos:

1 Alongamento de paravertebrais: postura de decúbito dorsal, flexão de membros inferiores (joelhos, quadril) e cabeça em direção ao tronco por 30 segundos, três repetições. 
2 Alongamento de paravertebrais: postura de decúbito dorsal, flexão de um membro inferior (joelho e quadril) em direção ao tronco e extensão do membro oposto por 30 segundos, três repetições para cada membro.

3 Alongamentos de abdutores: postura de decúbito dorsal, flexão de um membro inferior (joelho e quadril) cruzando sobre o membro oposto em extensão durante 30 segundos, três repetições para cada membro.

4 Alongamento de adutores: postura sentada com rotação externa de quadril, flexão de joelhos e planta dos pés em contato uma com a outra (borboleta). Manter a postura durante 30 segundos, três repetições.

5 Alongamento de isquiotibiais e paravertebrais: postura sentada com extensão de membros inferiores (joelhos), flexão de tronco e extensão de membros superiores (ombro e cotovelos) em direção aos pés. Manter a posição por 30 segundos, três repetições.

6 Alongamento de isquiotibiais: postura de decúbito dorsal com extensão de um membro inferior (quadril e joelho) e flexão do membro oposto (quadril) com extensão de joelho em direção ao tronco durante 30 segundos, três repetições.

Os exercícios foram realizados por um período de quatro meses, com frequência de uma vez por semana e duração de 30 minutos, constituindo desta forma, um protocolo com 16 sessões de fisioterapia.

\section{Anólise estatística}

Para análise estatística foi inicialmente elaborado um banco de dados no programa Microsoft Excel@ e os dados foram analisados por meio do programa Software Sigma-Stat ${ }^{\circledR} 3.5$. A normalidade dos dados foi verificada a partir do Teste de Shapiro Wilk, foi utilizado o teste " $\mathrm{t}$ " pareado para os dados normais e Teste de Wilcoxon para os não normais. Foram consideradas estatisticamente significativas as diferenças em que o valor $p$ foi menor que $5 \%(p<0,05)$.

\section{Resultados}

Diante da metodologia proposta neste estudo foi possível observar um aumento significativo da média da flexibilidade da musculatura posterior de membros inferiores dos participantes após intervenção fisioterapêutica $(p<0,0001$; $\mathrm{t}=12,95$ ) (Figura 1).

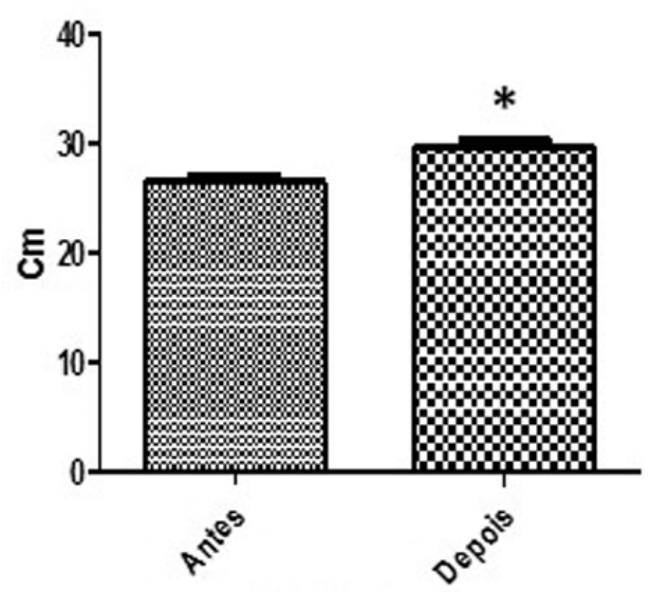

Flexibilidade

Figura l: Anólise estatística da média do grupo da flexibilidade da cadeia muscular posterior antes e após 16 sessões de fisioterapia em pacientes com PC. Legenda: ${ }^{*} p=0,0001 ; t=12,95$. Fonte: Do autor, 2018.

A tabela 2 apresenta os resultados individuais dos pacientes, demonstrando que houve melhora significativa na flexibilidade da cadeia muscular posterior de membros inferiores após 16 sessões de fisioterapia $(\mathrm{p}<0,05)$ em todos os seis indivíduos, com ganhos mínimos de $5,2 \mathrm{~cm}$ e máximos de $9,3 \mathrm{~cm}$.

\section{Discussão}

A utilização de exercícios físicos no processo de reabilitação de crianças com PC tem sido vastamente aplicada no cotidiano clínico de profissionais que atendem essa população devido à melhora da flexibilidade e espasticidade $^{19,20}$. Esses indivíduos apresentam diversas 
Tabela 2: Análise descritiva das médias pré e pós atendimento fisioterapêutico por paciente e em grupo

\begin{tabular}{c|c|c|c|c}
\hline Paciente & $\begin{array}{c}\text { MÉDIA } \\
\text { pré 1 }^{\mathrm{a}}(\mathrm{cm})\end{array}$ & $\begin{array}{c}\text { MÉDIA } \\
\text { pós 16 }{ }^{\mathrm{a}}(\mathrm{cm})\end{array}$ & $\begin{array}{c}\text { GANHOS } \\
(\mathrm{cm})\end{array}$ & $\begin{array}{c}\text { VALORES } \\
\text { DE } \mathrm{p}\end{array}$ \\
\hline 1 & 21,5 & 30,3 & 8,8 & $\begin{array}{c}\mathrm{P}<0.0001 ; \\
\mathrm{t}=5,718\end{array}$ \\
\hline 2 & 26,3 & 31,5 & 5,2 & $\begin{array}{c}\mathrm{P}<0.0001 ; \\
\mathrm{t}=6,186\end{array}$ \\
\hline 3 & 31,3 & 43,3 & 12 & $\begin{array}{c}\mathrm{p}<0,0001 ; \\
\mathrm{t}=7,462\end{array}$ \\
\hline 5 & 22,7 & 29,0 & 6,3 & $\begin{array}{c}\mathrm{p}<0,0001 ; \\
\mathrm{t}=7,462\end{array}$ \\
\hline 6 & 15,2 & 24,5 & 9,3 & $\begin{array}{c}\mathrm{p}<0,0001 ; \\
\mathrm{t}=5,409\end{array}$ \\
\hline MÉDIA & 24,1 & 32,2 & 8,1 & $\begin{array}{c}\mathrm{p}<0,0001 ; \\
\mathrm{t}=12,95\end{array}$ \\
\hline
\end{tabular}

Legenda: pré $1^{\mathrm{a}}$ (anteriormente à primeira sessão); pós $16^{a}$ (posteriormente à $16^{a}$ sessão). centímetros (cm). Valor de p significativo: $p<0,05$.

Fonte: Do autor, 2018.

características clínicas como as alterações neuromotoras, encurtamento muscular, alterações posturais e de marcha que frequentemente geram limitações e incapacidades na execução das atividades regulares ${ }^{14,15}$.

O objetivo deste trabalho foi avaliar os benefícios da fisioterapia para a melhora da flexibilidade da cadeia muscular posterior em crianças com PC espástica, por meio de alongamentos passivos, visto que este método de tratamento é de acessível aplicabilidade e com indicação nas alterações das funções motoras ${ }^{16,17}$.

Nossos resultados demonstraram que houve um aumento significativo da flexibilidade da cadeia muscular posterior dos pacientes logo após intervenção utilizando alongamentos passivos quer seja na avaliação individual quer seja em grupo. Na literatura científica, autores relatam que exercícios de alongamentos propiciam melhora imediata da flexibilidade, amplitude de movimento e espasticidade ${ }^{18-19}$.

O desenvolvimento das crianças com PC pode ser prejudicado devido à incapacidade motora, pois o movimento é considerado funda- mental para homem na exploração do ambiente onde vive ${ }^{22}$, é por meio da execução dos movimentos, que as habilidades motoras das crianças são desenvolvidas e estimuladas, tornando-as independentes e funcionais ${ }^{23}$.

Em nosso estudo também foi possível analisar um ganho de flexibilidade da cadeia muscular posterior em todos os seis pacientes, comparando-se a avaliação antes do início da intervenção com a anterior à $16^{\mathrm{a}}$ sessão de alongamentos, indicando, portanto, que o ganho de flexibilidade permaneceu ao longo do tempo e não apenas após cada sessão de fisioterapia. Achados semelhantes foram observados em uma pesquisa envolvendo a avaliação da flexibilidade de crianças PC diparéticas antes e após cinco sessões de hidroterapia, corroborando desta forma, com os nossos resultados ${ }^{20}$. Porém talvez, seja prudente termos cautela ao afirmarmos que o aumento da flexibilidade das crianças de ambos os estudos foi duradouro, uma vez que os mesmos não apresentaram em sua metodologia um período de follow up, a fim de acompanhar a resposta a longo prazo.

Entretanto, Moesch e colaboradores ${ }^{24}$ afirmam que os benefícios do alongamento dos músculos paravertebrais e isquiotibiais na melhora da flexibilidade da cadeia posterior podem ser observados mesmo após a interrupção dos exercícios por um período de oito semanas, visto que fáscias, ligamentos e cápsulas também apresentam melhora nos parâmetros da flexibilidade assim como no segmento muscular.

Um aspecto importante a considerar é a técnica de alongamento utilizado. Pesquisas utilizando o alongamento estático, ativo ou passivo como intervenção para escolares sem comprometimento motor, apontam que o alongamento estático ideal deve ser realizado de forma que os músculos atinjam sua amplitude máxima e assim mantê-los nessa posição por tempo suficiente, pelo menos 15 a 20 segundos, a fim de estimular tendões e fibras musculares, de modo que a resistência muscular diminua e permita o estiramento necessário ${ }^{25,26}$. O tempo do alongamento empregado pelos autores cita- 
dos acima e o do estudo em questão estão em concordância quando o objetivo for melhora da flexibilidade.

Sugere-se que durante a lesão que levou à PC, ocorra um comprometimento dos circuitos neurais que modulam o tônus, alterando as propriedades elétricas intrínsecas dos neurônios, ou ainda, que alterações estruturais permanentes nas propriedades mecânicas intrínsecas dos tecidos musculares podem ter levado ao aumento do tônus. Sendo assim, vale ressaltar que a hipertonia é uma alteração importante em algumas pessoas com PC e é um fator limitante para a execução de movimentos voluntários e funcionais, e que a mesma pode ser atenuada com técnicas manuais como dissociação de cinturas, mobilizações, e também com alongamentos ${ }^{27}$. Esse, portanto, foi o método escolhido para a realização deste estudo, e pode-se inferir que os alongamentos, quando realizados na forma estática e passiva, leva à uma melhora da flexibilidade dos indivíduos com PC estudados. Em conformidade com a presente pesquisa, outros estudos destacam ainda a importância e os benefícios da técnica de alongamento para algumas alterações e padrões posturais específicos nas crianças com PC, como o equinismo dos pés que é tratado com alongamento de tríceps sural na postura em decúbito dorsal, com o joelho em extensão e o tornozelo em plantiflexão máxima ${ }^{19}$.

Embora tenhamos encontrado limitações neste estudo, como a dificuldade de formar um grupo homogêneo com uma amostra maior, que permitiria randomizar os participantes e formar um grupo controle, esta pesquisa contriuirá para reforçar e complementar a literatura acerca dos tratamentos propostos na PC, e busca embargar uma maior valorização da técnica de cinesioterapia, por meio do alongamento passivo, como recurso utilizado para melhora da flexibilidade desse grupo de paciente. Infere-se ainda que, com a melhora da flexibilidade possa haver ganhos motores e na funcionalidade deste indivíduos.

\section{Conclusões}

Este estudo infere que a fisioterapia por meio de exercícios de alongamentos passivos propicia melhora da flexibilidade da cadeia muscular posterior de crianças com PC, devido ao relaxamento e diminuição do tônus muscular. Este ganho de flexibilidade muscular pode, portanto melhorar a qualidade de vida e funcionalidade dos pacientes tratados por esta modalidade de reabilitação.

\section{Referências}

1. Rosenbaum P, Paneth N, Leviton A, Goldstein M, Bax M, Damiano D, et al. A report: the definition and classification of cerebral palsy April 2006. Developmental Medicine and Child Neurology. 2007;49(2):8-14.

2. Daher MT, Martins PLS, Esperidião AP, Felisbino Júnior P, Nascimento VN, Pereira Júnior JH, et al. Relationship between function and presence and type of deformity in patients with cerebral palsy. Coluna/Columna. 2016;15(4): 275-8.

3. Chiarello LA, Palisano RJ, Bartlett DJ, McCoy SW. A multivariate model of determinants of change in gross motor abilities and engagement in self-care and play of young children with cerebral palsy. Physical \& Occupational Therapy In Pediatrics. 2011;31:150-68.

4. Himpens E, Van den Broeck C, Oostra A, Calders P, Vanhaesebrouck P. Prevalence, type, distribution, and severity of cerebral palsy in relation to gestational age: a meta-analytic review. Developmental Medicine and Child Neurology. 2008;50(5):334-40.

5. Hoffmann RA, Tafner MA, Fischer J. Paralisia cerebral e aprendizagem: um estudo de caso inserido no ensino regular. Revista do Instituto Catarinense de Pós-graduação. 2016;2(12):1-15.

6. Cans C, Dolk H, Platt MJ, Colver A, Prasausklene A, Rägeloh-Mann IK. Recommendations from the SCPE collaborative group for defining and classifying cerebral palsy. Developmental Medicine and Child Neurology. 2007;49(s109):35-8. 
7. Bartlett DJ, Chiarello LA, Mccoy SW, Palisano RJ, Jeffries L, Fiss AL, Rosenbaum P et al. Determinants of gross motor function of young children with cerebral palsy: a prospective cohort study. Developmental Medicine \& Child Neurology. 2016;56:275-82.

8. Leite MRS, Prado GF. Paralisia cerebral: aspectos fisioterapêuticos e clínicos. Revista Neurociências. 2004;12(1):41-5.

9. Zanini G, Cemin NF, Peralles SN. Paralisia Cerebral: causas e prevalências. Revista Fisioterapia em Movimento. 2009;22(3):375-81.

10. Guimarães CL, Pizzolatto TCO, Coelho ACS, Freitas STT. Aspectos clínicos epidemiológicos de crianças com paralisia cerebral assistidas pela clínica escola de Fisioterapia UNIP-São José dos Campos. Journal of the Health Sciences Institute. 2014;32(3):281-5.

11. Brasil. Ministério da Saúde. Secretaria de Atenção à Saúde. Departamento de Ações Programáticas Estratégicas. Diretrizes de atenção à pessoa com paralisia cerebral / Ministério da Saúde, Secretaria de Atenção à Saúde, Departamento de Ações Programáticas Estratégicas. - Brasília : Ministério da Saúde, 2013. 80 p.

12. Assis-Madeira EA, Carvalho SG, Blascovi-Assis SM. Desempenho funcional de crianças com paralisia cerebral de níveis socioeconômicos alto e baixo. Revista Paulista de Pediatria. 2013;31(1):51-7.

13. Ferreira LSM, Santos VB, Pessoa PB, Silva OS, Sousa SMB, Silva L. M. Análise dos Fatores de Risco Para Desenvolvimento de Encefalopatia Crônica Não Progressiva. Revista Brasileira de Ciências da Saúde. 2015;19(2):129-34.

14. Curtis DJ, Butler P, Saavedra S, Bencke J, Kallemose $\mathrm{T}$, Sonne- Holm S, et al. The central role of trunk control in the gross motor function of children with cerebral palsy: a retrospective cross-sectional study. Developmental Medicine and Child Neurology. 2015;57(4):351- 7.

15. Park EY, Kim WH. Structural equation modeling of motor impairment, gross motor function, and the functional outcome in children with cerebral palsy. Research In Developmental Disabilities. 2013;34:1731-9.

16. Smania N, Picelli A, Munari D, Geroin C, Ianes P, Waldner A, et al. Rehabilitation procedures in the management of spasticity. European Journal of Physical and Rehabilitation Medicine. 2010;46(3):423-38.
17. Dalesse ACE, Grecco LAC, Oliveira CS, Golin MO. Efeitos de técnicas fisioterapêuticas para reduzir a hipertonia em crianças com paralisia cerebral. Rev Terapia Manual Posturologia. 2013;11(51):100-4.

18. Pinheiro IM, Góes ALB. Efeitos imediatos do alongamento em diferentes posicionamentos. Fisioterapia em Movimento. 2010;23(4):593-603.

19. Oliveira LS, Golin, MO. Técnica para redução do tônus e alongamento muscular passivo: efeitos na amplitude de movimento de crianças com paralisia. ABCS Health Sciences. 2017;42(1):27-33.

20. Espindula AP, Jammal MP, Guimarães CSO, Abate DTRS, Reis MA, Teixeira VPA. Avaliação da flexibilidade pelo método do Flexômetro de Wells em crianças com Paralisia Cerebral submetidas a tratamento hidroterapêutico: estudo de casos. Acta Scientiarum. Health Sciences. 2010;32(2):163-7.

21. Ribeiro MF, Patrizzi LJ, Teixeira VPA, Espindula AP. Equilibrium and muscle flexibility in elderly people subjected to physiotherapeutic intervention. Acta Scientiarum. Health Sciences. 2016;38(2):129-36.

22. Zonta MB, Ramalho Junior A, Santos LHC. Avaliação funcional na paralisia cerebral. Acta Pediátrica Portuguesa. 2011;42(1):27-32.

23. Santos PD, Silva FC, Ferreira EG, Iop RR, Bento GG, Silva R. Instrumentos que avaliam a independência funcional em crianças com paralisia cerebral: uma revisão sistemática de estudos observacionais. Fisioterapia e Pesquisa. 2016;23 (3):318-28.

24. Moesch J, Mallmann S, Tomé F, Vieira L, Ciqueleiro RT, Bertolini GRF. Effects of three protocols of hamstring muscle stretching and paravertebral lumbar. Fisioterapia em Movimento. 2014;27(1):85-92.

25. Moreira RFC, Akagi FH, Wun PYL, Moriguchi CS, Sato TO. Effects of a school based exercise program on children's resistance and flexibility. Work. 2012;41:922-8.

26. Mayorga-Vega D, Merino-Marban R, SánchezRivas R, Viciana J. Effect of a short-term static stretching training program followed by five weeks of detraining on hamstring extensibility in childen aged 9-10 years. Journal of Physical Education Sport. 2014;14(3):355-9.

27. Gomes C, Golin M. Tratamento Fisioterapêutico Na Paralisia Cerebral Tetraparesia Espástica, Segundo Conceito Bobath. Revista Neurociências. 2013;21(2):278-85. 